\title{
A Facile Strategy to Prepare Smart Coating with Autonomous Self-Healing and Self-Reporting
}

\section{Functions}

Shusheng Chen, ${ }^{\dagger}$ Ting Han, ${ }^{\star}{ }^{\star}$ Ying Zhao, ${ }^{\dagger}$ Wenjun Luo, ${ }^{\dagger}$ Zhong Zhang, ${ }^{\prime}$ Haibin Su, ${ }^{\S}$ Ben Zhong Tang, ${ }^{*},{ }^{\prime} \S$ and Jinglei Yang ${ }^{*,+}$

'Department of Mechanical and Aerospace Engineering and ${ }^{\S}$ Department of Chemistry, The Hong Kong University of Science and Technology, Clear Water Bay, Hong Kong SAR, China

${ }^{\ddagger}$ Center for AIE Research, College of Materials Science and Engineering, Shenzhen University, Shenzhen 518060, China

"CAS Key Laboratory of Nanosystem and Hierarchical Fabrication and CAS Center for Excellence in Nanoscience, National Center for Nanoscience and Technology, Beijing 100190, China 


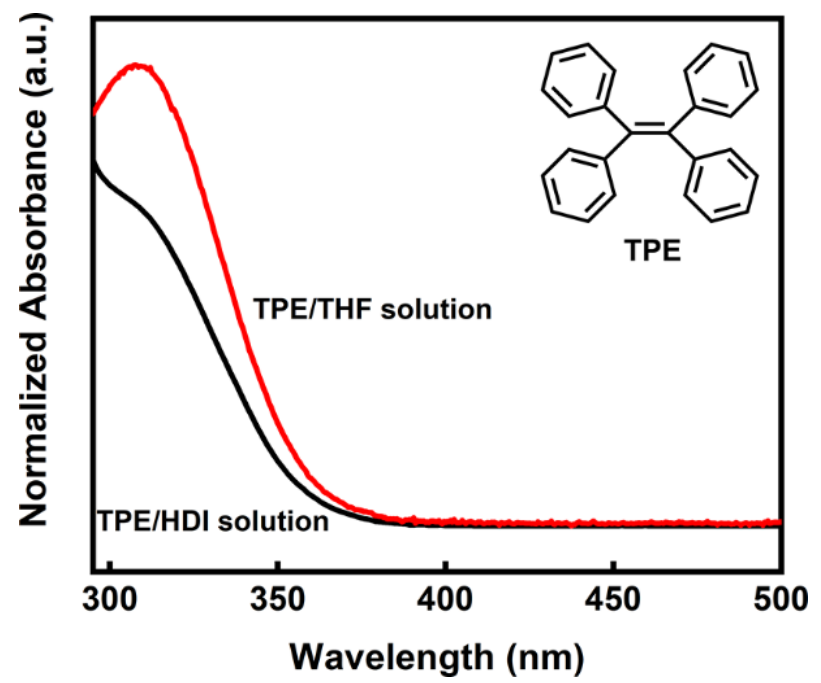

Figure S1. UV-vis absorption spectra of TPE/HDI and TPE/THF solution. The insert is the chemical structure of TPE.

During the isothermal process at $180^{\circ} \mathrm{C}$ for $1 \mathrm{~h}$, the HDI core of microcapsules was completely evaporated. Typically, the weight loss of microcapsules was $66.5 \mathrm{wt} \%$, which includes the total liquid core and partial shell materials. The partial shell materials took $13.3 \mathrm{wt} \%$ of the whole shell materials from the related TGA curve (Figure S2). Thus, the core fraction of microcapsules could be calculated according to the following equations ${ }^{1}$ :

$$
\begin{aligned}
& C_{\text {core }}+C_{\text {shell }}=C_{\text {microcapsules }} \\
& C_{\text {shell }} /\left(1-C_{\text {core }}\right)=13.3 \mathrm{wt} \%
\end{aligned}
$$

The weight loss of microcapsules, their HDI core and shell during the isothermal process were defined as $\mathrm{C}_{\text {microcapsules}}, \mathrm{C}_{\text {core }}$ and $\mathrm{C}_{\text {shell }}$, respectively. The core factions of microcapsules as a function of agitation rate for PU synthesis and reaction duration for PUF synthesis were calculated and summarized in Table S1 and S2 and Figure S3. 


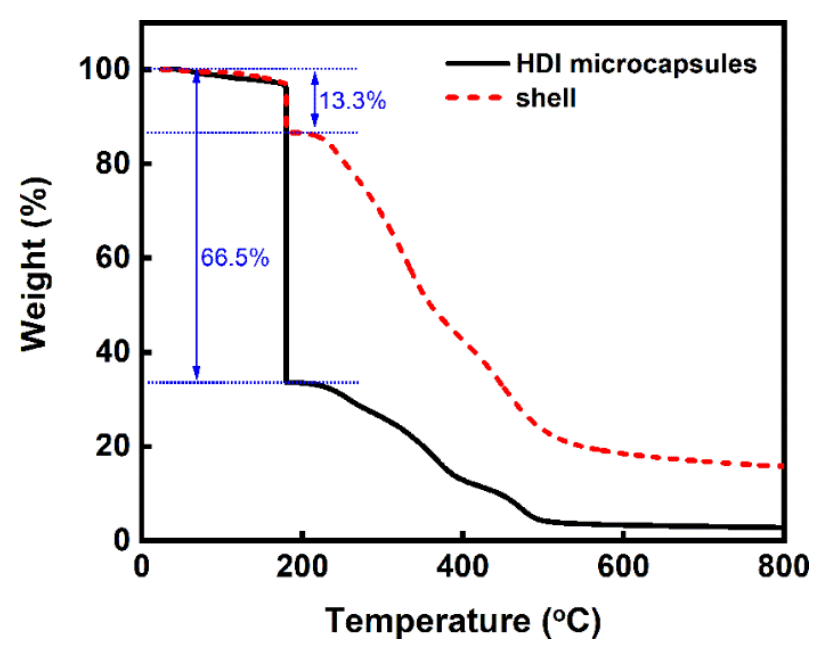

Figure S2. Typical TGA curves of HDI microcapsules and shell (1000 rpm of agitation rate for PU synthesis and $1 \mathrm{~h}$ of reaction duration for PUF synthesis). The samples were first heated at a rate of $20^{\circ} \mathrm{C} / \mathrm{min}$ from 25 to $180^{\circ} \mathrm{C}$ and then kept isothermal for $1 \mathrm{~h}$ before the samples were finally heated to $800{ }^{\circ} \mathrm{C}$ under a nitrogen atmosphere.

Table S1. Effect of agitation rate of PU synthesis on the size and core fraction of microcapsules ${ }^{a}$

\begin{tabular}{cccccc}
\hline & No. 1 & No. 2 & No. 3 & No. 4 & No. 5 \\
\hline Agitation rate $(\mathrm{rpm})$ & 600 & 800 & 1000 & 1200 & 1400 \\
Average diameter $(\mu \mathrm{m})^{b}$ & $313 \pm 128$ & $215 \pm 39$ & $106 \pm 17$ & $64 \pm 19$ & $45 \pm 13$ \\
Core fraction $(\mathrm{wt} \%)^{c}$ & $77.1 \pm 3.2$ & $73.4 \pm 1.5$ & $61.7 \pm 3.7$ & $55.8 \pm 2.6$ & $46.2 \pm 1.7$ \\
\hline
\end{tabular}

${ }^{a}$ The inner polyurea (PU) shells of the microcapsules were synthesized through interfacial polymerization. The agitation rate of the interfacial polymerization was controlled and its effect on the size and core fraction of microcapsules was studied. The reaction of the PU microcapsules with UF prepolymer lasted for $1 \mathrm{~h}$. ${ }^{b}$ The size of the microcapsules was analyzed from SEM images. ${ }^{c}$ The core fractions of microcapsules were calculated from the isothermal TGA results.

Table S2. Effect of reaction duration of PUF synthesis on the core fraction of microcapsules ${ }^{a}$

\begin{tabular}{lcccccc}
\hline & No. 1 & No. 2 & No. 3 & No. 4 & No. 5 & No. 6 \\
\hline Reaction duration (h) & 0.5 & 1 & 2 & 3 & 4 & 5 \\
Core fraction (wt\%) $^{b}$ & $68.5 \pm 2.4$ & $61.7 \pm 3.7$ & $53.4 \pm 4.6$ & $46.2 \pm 1.8$ & $43.8 \pm 2.4$ & $38.6 \pm 1.5$ \\
\hline
\end{tabular}


${ }^{a}$ The poly(urea-formaldehyde) (PUF) shells were deposited on the PU microcapsules by in situ polymerization. The agitation rate of the interfacial polymerization was chosen as $1000 \mathrm{rpm}$. The reaction time of the PU microcapsules with UF prepolymer in the presence of resorcinol in EMA aqueous solution was controlled and its effect on the core fraction of microcapsules was studied. ${ }^{b}$ The core fractions of microcapsules were calculated from the isothermal TGA results.
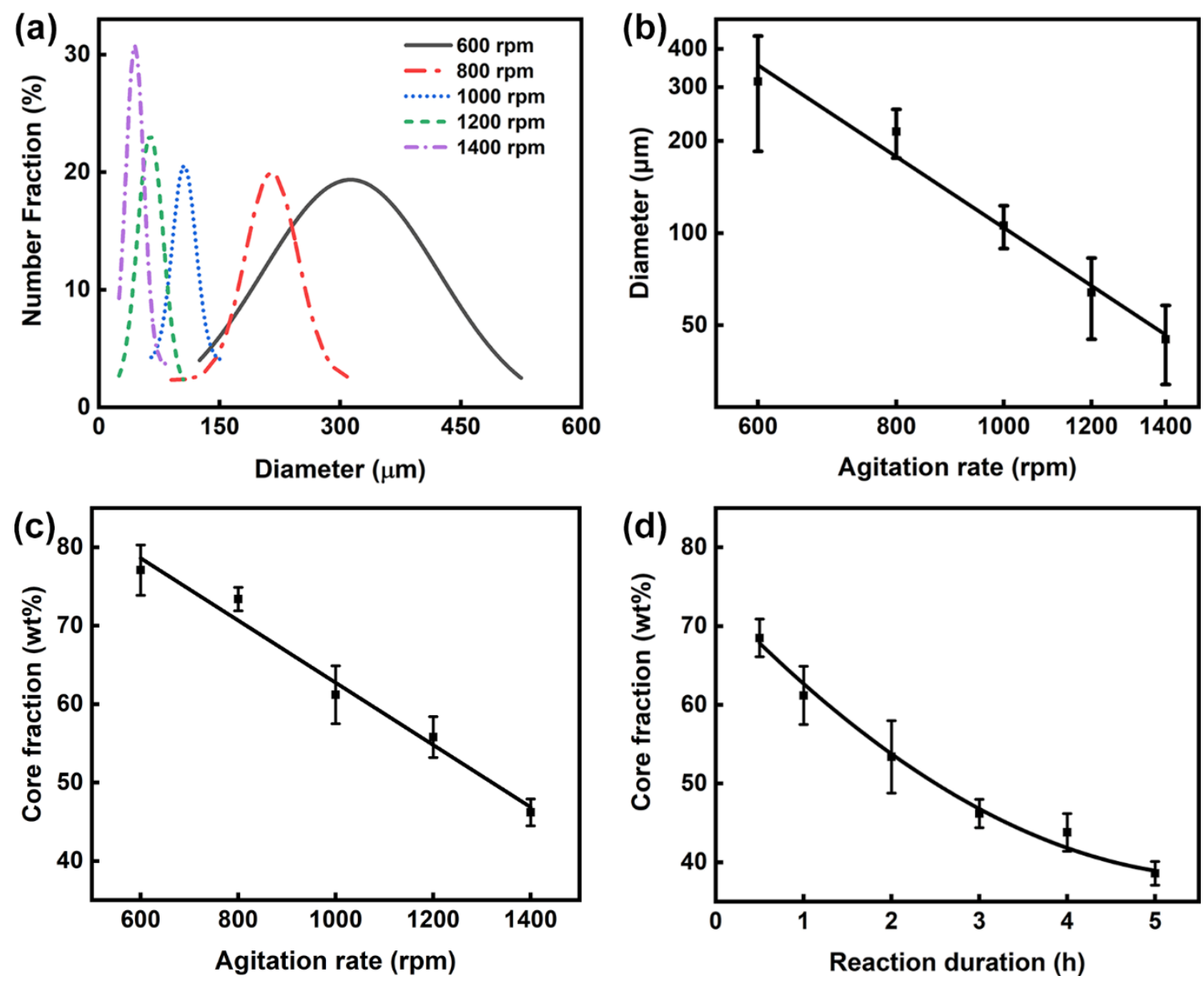

Figure S3. (a) Size distributions, (b) diameter, and (c) core fraction of microcapsules as a function of agitation rate for PU synthesis. (d) Core fraction of microcapsules as a function of reaction duration for PUF synthesis. 

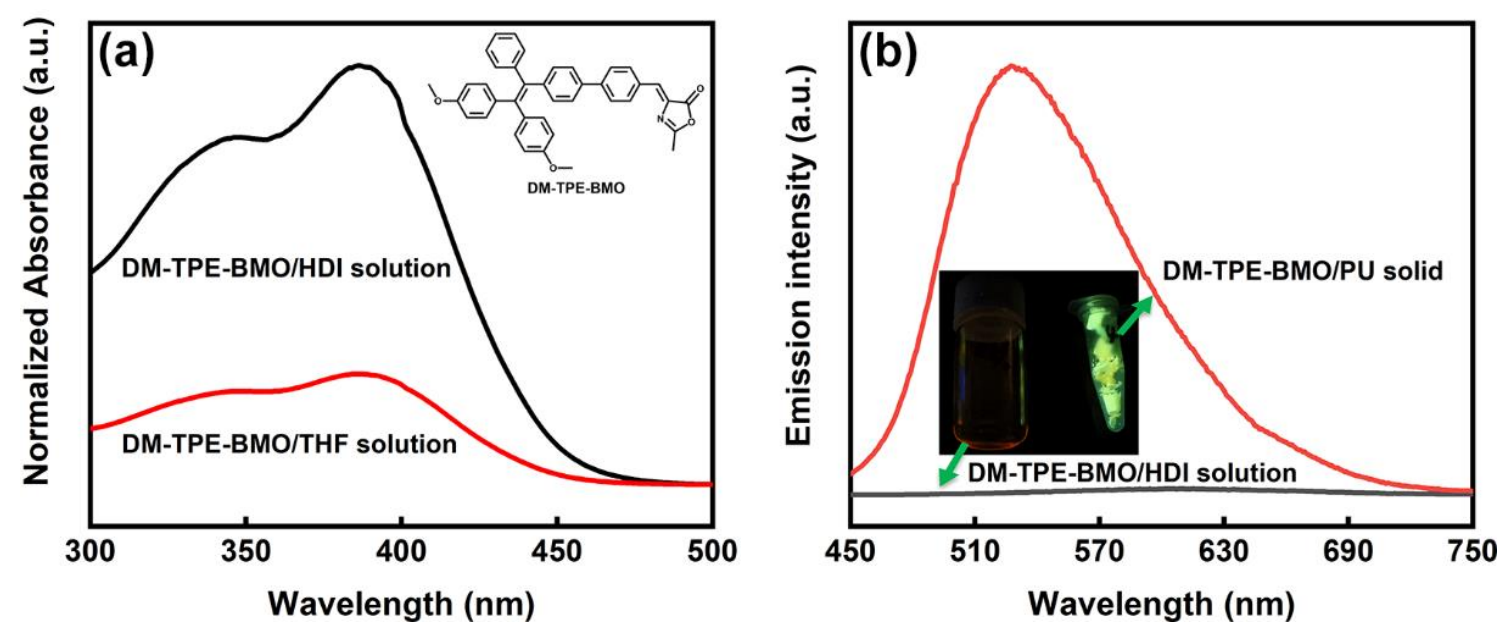

Figure S4. (a) UV-vis absorption spectra of DM-TPE-BMO/HDI and DM-TPE-BMO/THF solutions. The inset is the chemical structure of DM-TPE-BMO. (b) PL spectra of DM-TPEBMO/HDI solution and DM-TPE-BMO/PU solid. Excitation wavelength: $380 \mathrm{~nm}$. The inset is photograph of DM-TPE-BMO/HDI solution and DM-TPE-BMO/PU solid taken under the illumination of UV light. 

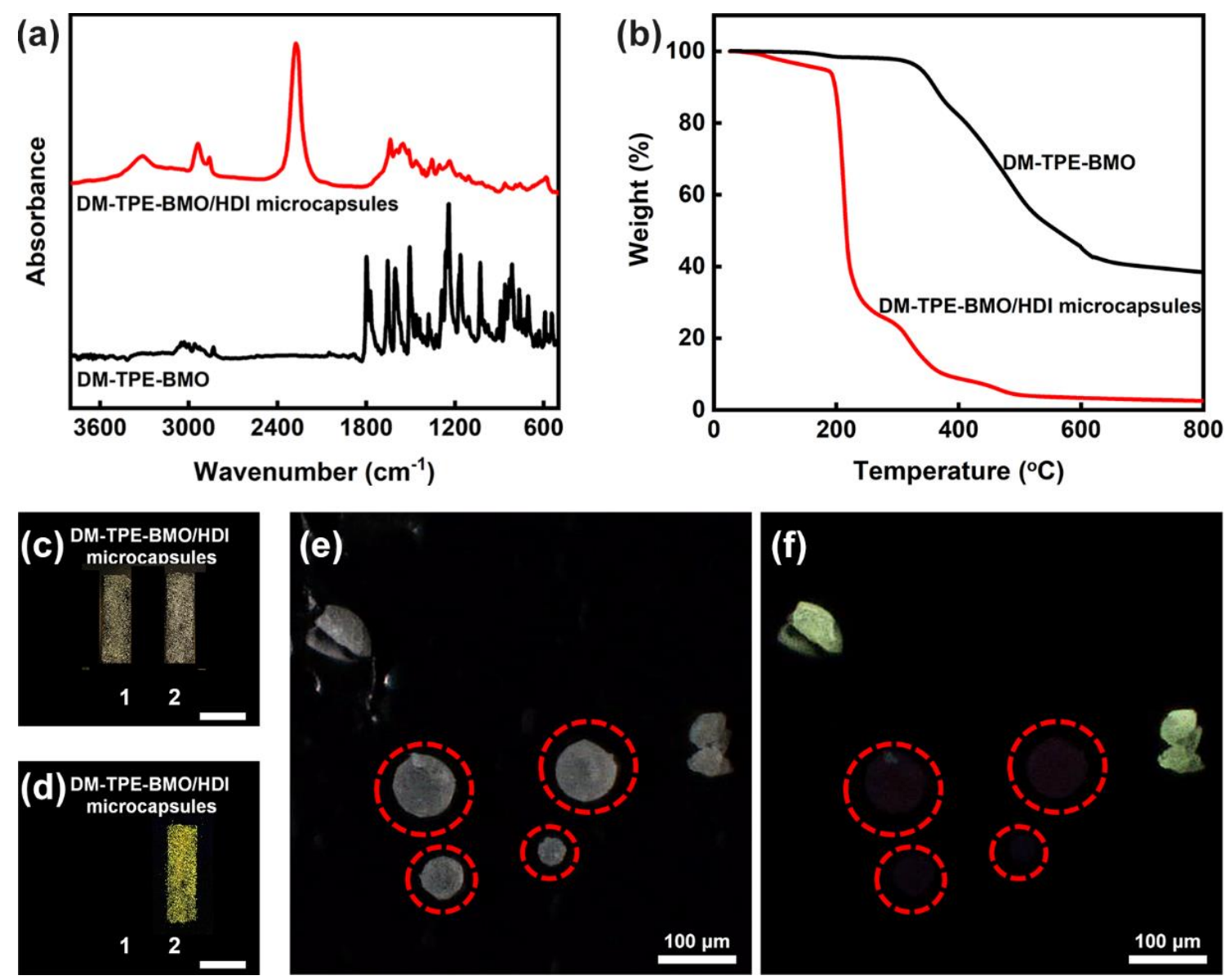

Figure S5. (a) FTIR spectra and (b) TGA curves of DM-TPE-BMO/HDI microcapsules and pure DM-TPE-BMO. (c and d) Photographs of DM-TPE-BMO/HDI microcapsules taken under the illumination of (c) white light and (d) UV light. The microcapsules on strip no.1 are intact, whereas those on strip no. 2 are ruptured. The white scale bar indicates $25 \mathrm{~mm}$ in both images. (e-f) Micrographs of DM-TPE-BMO/HDI microcapsules taken under the illumination of (e) white light and (f) UV light.

\section{REFERENCES}

(1) Sun, D.; Zhang, H.; Tang, X.-Z.; Yang, J. Water resistant reactive microcapsules for selfhealing coatings in harsh environments. Polymer 2016, 91, 33-40. 